\title{
PURITY AND INTENSITY OF MONOCHROMATIC LIGHT SOURCES.
}

By P. G. Nutting.

Spectrum lines differ greatly in spectral width or purity even when produced under similar conditions. An increase in intensity from any cause or an increase in density of the radiating gas always causes an increase in width or decrease in purity of a line. The choice of a monochromatic source for a given piece of work will depend upon(I) the maximum of purity and mimimum of intensity required for the work in hand, (2) the conditions of production under which a given line will be as bright as possible for a given width, and (3) upon what lines, under given conditions, will afford a minimum of impurity with a maximum of intensity.

In polarimetry, for example, great intensity is required, while excessive purity is not essential in measuring ordinary rotations. Interferometry, on the other hand, requires excessive purity, but only a moderate intensity. This paper is devoted to a consideration of conditions (2) and (3) under which a maximum ratio of intensity to purity may be obtained.

A. A. Michelson ${ }^{1}$ and F. L. O. Wadsworth, ${ }^{2}$ from the "visibilty" of lines in the interferometer, calculated their width and established relations between spectral width and atomic weight and pressure. This method of determining width is free from corrections for slit width and resolving power, but involves considerable uncertainty in the matter of difference in path or time phase at the source. With both the Michelson and Fabry-Pérot interferometers the fainter components of composite lines are easily overlooked and ignored.

${ }^{1}$ Phil. Mag., (5) 34, p. 2So-299; I892.

${ }^{2}$ Astrophysical Journal, 6, p. 30 ; 1897. 
Many lines are included in the published data of Michelson and Wadsworth which are known to be composite. The author ${ }^{3}$ has measured the width of lines directly with an echelon provided with a micrometer eyepiece. Corrections for slit width and resolving power were determined experimentally and applied. With the echelon the character of a composite line is at once evident.

\section{THE THEORY OF SPECTRAL PURITY.}

The mathematical definition of the spectral purity of a light source is more or less arbitrary. For instance, the total width of a line in wave length units might be taken as a measure of purity. This would be analogous to an ordinary length standard defined as the distance between two scratches on a bar. If one of the scratches is a broad groove, the length is uncertain by the width of this groove. Suppose the green cadmium light waves magnified to the length of such a meter bar. Then one of the limits would be three scratches, the outer ones o.or $\mathrm{mm}$ apart. On this basis the light is pure or the length is certain to one part in roo,ooo. On a similar basis sodium yellow is pure, at moderate intensity, to one part in about 700 , mercury green to one part in I0,000, helium yellow to one part in 200,000 or one part in I 5,000 , if the satellite is included.

Such a definition of purity does very well, perhaps, for the sodium yellow, but is hardly fair in case the impurity consists of faint distant satellites. Obviously every component or component element should be weighted according to its intensity. Such a definition of purity leads mathematically to an expression in which impurity is analogous to the mean square error in the theory of errors and to the radius of gyration in mechanics. Hence corresponding to the center of mass in the latter case is a mean wave length which may be called the center of luminosity. Call this center of luminosity $\lambda_{c}$ and represent by $E$ the intensity of a component of wave length $\lambda$. Then

$$
\lambda_{\mathrm{c}} \equiv \frac{E_{1} \lambda_{1}+E_{2} \lambda_{2}+\ldots}{E_{1}+E_{2}+\ldots}=\frac{\int \lambda d E}{\int d E}
$$

\footnotetext{
${ }^{3}$ Astrophysical Journal, 24, p. III-I24; 1906.
} 
by definition. The impurity $I$ is defined by

$$
\begin{gathered}
I^{2} \equiv \frac{E_{1}\left(\lambda_{1}-\lambda_{\mathrm{c}}\right)^{2}+E_{2}\left(\lambda_{2}-\lambda_{\mathrm{c}}\right)^{2}+\ldots}{E_{1}+E_{2}+\ldots} \\
=\frac{\int\left(\lambda-\lambda_{\mathrm{c}}\right)^{2} d E}{\int d E}
\end{gathered}
$$

expressed in wave length units. It is frequently preferable to use a pure number, the specific impurity, which we may call $I_{\mathrm{s}}$ and define as

$$
I_{\mathrm{s}}=I \lambda_{\mathrm{c}}
$$

Consider now the impurity of sodium yellow on this basis. For a pair of lines

$$
I=\left(\lambda_{1}-\lambda_{2}\right) \frac{\sqrt{E_{1} E_{2}}}{E_{1}+E_{2}}=\left(\lambda_{1}-\lambda_{2}\right) \frac{K^{\frac{1}{2}}}{\mathrm{I}+K}
$$

where $K \equiv E_{1}: E_{2}$, the ratio of the intensities of the two lines. For sodium yellow $K=\mathrm{I} .6, \lambda_{1}=5890.2, \lambda_{2}=5896.2$, hence $I=$ $6.0 \sqrt{\mathrm{I} .6}: 2.6=2.9 \mathrm{t}-\mathrm{m}$. The specific impurity is $2.9: 5892.5=0.00049$ or about one part in two thousand.

For a single line bounded by the limiting wave lengths $\lambda_{1}$ and $\lambda_{2}$ and uniform intensity throughout, $d E=h d \lambda$,

$$
\begin{gathered}
I^{2}=\frac{\int_{\lambda_{1}}^{\lambda_{2}}\left(\lambda-\frac{1}{2}\left(\lambda_{1}+\lambda_{2}\right)\right)^{2} h d \lambda}{\int_{\lambda_{1}}^{\lambda_{2}} h d \lambda} \\
I=\frac{\lambda_{2}-\lambda_{1}}{2 \sqrt{3}}=0.2887 \delta
\end{gathered}
$$

where $\delta$ is the width of the line or band in wave lengths. A green line at 5500 measuring $0.055 \mathrm{t}-\mathrm{m}$ broad is thus impure to the extent of $0.016 \mathrm{t}-\mathrm{m}$ or to one part in three million. A complete continuous spectrum of constant energy throughout would have infinite impurity.

If the distribution of energy in a line or band is a cosine function throughout a total width $\delta$, the impurity may be shown to be $0.2 \mathrm{I} 7 \delta$. 
Normal spectrum lines appear to possess an energy distribution corresponding roughly to the probability function

$$
E=A e^{-a\left(\lambda-\lambda_{e}\right)^{2}}
$$

where $E$ is the energy per unit wave length.

This gives an integrable form for the impurity which comes out in this case simply

$$
I=(2 a)^{-\frac{1}{2}}
$$

This case is of extreme importance. It represents not only most normal lines and many transmission bands but the luminosity curve of white light as well. If the distribution of energy throughout a spectrum is uniformly constant, then viewed by a mean normal eye, the distribution of luminosity is given by

$$
L=L_{\mathrm{o}} e^{-\pi\left(\lambda-\lambda_{\mathrm{e}}\right)^{2}}
$$

$\lambda$ being expressed in seventh-meters and $L$ in light units per unit wave length. Hence the luminous impurity of "white" light is I $\sqrt{2 \pi}=0.399 \mathrm{~s}-1 \mathrm{n}$, and the specific luminous impurity is 0.08 .

It is of interest to compare the impurities of lines of the above three types of structure having the same total energy and the same specific intensity at their centers, that is lines whose graphs have the same area and the same height.

Since in the three cases

$$
E_{1}=\operatorname{const}(\equiv h), \quad E_{2}=h \cos \left(\lambda-\lambda_{\mathrm{c}}\right), E_{3}=h e^{-a\left(\lambda-\lambda_{\mathrm{c}}\right)^{2}}
$$

the condition of equal total energies gives

$$
\delta_{1}=\frac{2}{\pi} \delta_{2}=\sqrt{\frac{\pi}{a}}
$$

$\delta_{1}$ being the width of the line of constant intensity throughout and $\delta_{2}$ that of the line having a cosine distribution. The impurities in the three cases being as deduced above

$$
0.288 \delta_{1}, \quad 0.2 \mathrm{I} 7 \delta_{2}, \quad(2 a)^{-\frac{1}{2}}
$$

they are in the proportion

$$
\text { I, } \quad 0.759, \quad \text { I.382. }
$$


The spectral width of a line of the third type measured visually is, however, quite different from the width $(\pi: \alpha)^{\frac{1}{2}}$ given by equivalent energy. The visual impurity of lines of this type will be taken up later on.

\section{ACTUAL AND VISUAL LINE WIDTH AND STRUCTURE}

The brighter portions of a line or band always appear relatively less bright than they really are, for by Fechner's law the visual sensation of brightness increases with the logarithm of the actual luminosity. Hence the spectral curve of brightness is always flatter than the curve of luminosity and becomes zero when the intensity reaches the threshold value for the retina and wave length in question. Hence it is evident that impurity of radiation, luminous impurity, and visual impurity are in general quite different from one another. Further, a line may appear visually sharp and well defined, permitting of an accurate measurement of a definite width, even though its energy curve has wide, diffuse wings.

For example, consider an ordinary normal line whose energy curve $E(\lambda)$ is

$$
E=E_{\mathrm{m}} e^{-\alpha\left(\lambda-\lambda_{\mathrm{c}}\right)^{2}}
$$

Its luminosity curve $L(\lambda)$ is $L=E: E_{\text {o }}$ or

$$
L=\frac{E_{\mathrm{m}} e^{-\alpha\left(\lambda-\lambda_{\mathrm{c}}\right)^{2}}}{E_{\mathrm{o}}}
$$

$E_{\mathrm{m}}$ being the intensity of the line (in watts per unit wave length) at its center and $E_{\mathrm{o}}$ the threshold value of the observer's eye for the wave length in question. Hence the curve of visual brightness $B(\lambda) \equiv \log L$ is

$$
B=\log \left(E_{\mathrm{m}} \cdot E_{\mathrm{o}}\right)-a\left(\lambda-\lambda_{\mathrm{c}}\right)^{2},
$$

a parabola axial on the axis of ordinates. Hence such a normal line will have a central maximum $\log \left(E_{\mathrm{m}}: E_{\mathrm{o}}\right)$ and a definite apparent width

$$
\delta \lambda=2\left(\frac{\log \left(E_{\mathrm{m}}: E_{\mathrm{o}}\right)}{a}\right)^{\frac{2}{2}}
$$


extending from $B=0$ on one side of the center to $B=0$ on the other side. The first derivative of $B$ has a large value, namely $\pm 2\left(a \log E_{\mathrm{m}}: E_{\mathrm{o}}\right)^{\frac{1}{2}}$ at the apparent edges of the line, hence such lines appear sharply defined.

It has been customary, in measuring the width of lines, to tacitly assume that the exponential energy curve is identical with or at least proportional to the curve of visual brightness. The resultant error is of slight consequence on rough work but in precise measurement assumes vital significance.

Analogous to radiant and luminous impurity, the visual impurity may be defined as the square root of

$$
\frac{\left.\int-\lambda_{\mathrm{c}}\right)^{2} d B}{\int d B}
$$

in which the visual brightness $B(\lambda)$ is the logarithm of the luminosity $L(\lambda)$, which in turn is the ratio of the spectral energy $E(\lambda)$ to the threshold value $E_{\mathrm{o}}(\lambda)$. Taking the above used exponential form for the energy curve of a line, its visual impurity as above defined may be calculated to be

$$
I=\frac{1}{\sqrt{5}}\left(\frac{\log \left(E_{\mathrm{m}} \cdot E_{\mathrm{o}}\right)}{a}\right)^{\frac{1}{2}}
$$

which is 0.223 of the (apparent) width of the line $\delta \lambda$.

\section{NORMAL AND COMPOSITE LINES.}

Classified according to structure and behavior, spectrum lines fall into two quite distinct classes, which may be distinguished as normal and composite. Normal lines are single and narrow when from a feebly excited source and with increasing intensity broaden continuously, reversing or doubling at some intermediate intensity. Composite lines consist of aggregates of other lines more or less fixed in position and relative intensity called primary and satellites but lying so close together as not to be resolvable with a prism or grating.

Composite lines are of many types, characterized by the form and variability of their components. The simplest are perhaps the yellow and blue helium lines. These have each a single companion line about half a tenth-meter removed from the primary on the side 
toward the red, fixed in position and relative intensity. The yellow copper lines $\lambda 5700$ and $\lambda 5782$ are each usually double but sometimes triple. With increase in intensity, each component broadens in situ instead of retreating from its neighbor, as would be the case if the doubling or tripling were mere normal reversal. Triple lines are numerous in the spectral of cadminm, zinc, and related metals, component lines remaining fixed in position and relative intensity. The green thallium line divides unsymmetrically at one stage of its development, following which the broader component divides again, so that at one stage this line is triple in structure with all three components variable. The yellow sodium lines vary in a similar manner, becoming very complex before reversal proper occurs, the components blurring one another by overlapping to sucli an extent that it is difficult to tell just what does occur.

The seven chief visible mercury lines are all composite and all different in structure. They have been very successfully photographed by Janicki. ${ }^{4}$ Extreme types are $\lambda 4047$ consisting of four equidistant bands of about equal intensity and $\lambda 546 \mathrm{I}$ consisting of a strong primary with five faint satellites; two of these five are diffuse and very variable in position and relative intensity while the other three are extremely sharp and fixed in position, though variable in relative intensity.

These illustrations of compound structure indicate that its structure and variability should be carefully studied before a composite line is used at all as a monochromatic source. Only when its satellites are relatively faint and fixed or else very near the primary is a composite line to be used as a simple line. Let us calculate the impurity as above defined of $\mathrm{Hg} \lambda 4047$.

Impurity of $\mathrm{Hg} 4047$

$\begin{array}{lcccc}\text { Components } & -0.05 & \text { primary } & +0.06 & \text { +0.I2 t } \mathrm{t}-\mathrm{m} \text {. } \\ \text { Intensity } & 6 & \text { IO } & 6 & 4\end{array}$

Impurity $=\left(\Sigma E\left(\lambda-\lambda_{\mathrm{c}}\right)^{2}: \Sigma E\right)^{\mathrm{T} / 2}=0.065 \mathrm{t}-\mathrm{m}$.

Specific impurity $0.0654047=0.000016$ or about two parts in I00,000-an impurity prohibitive for the best work. With the green mercury line the case is different.

${ }^{4}$ Ludwig Janicki: Inaugural Dissertation, Halle, 1905.

$16360-07-9$ 
Impurity of $\mathrm{Hg} 5461$.

Components $-0.09-0.06$ primary +0.II +0.I3 +0.22 Intensity 240 8 2

Impurity (neglecting the width of the primary) $0.0067 \mathrm{t}-\mathrm{m}$.

Specific impurity 0.00000125 , or roughly one part in a million. This is sufficiently small to permit of the use of this line in interferometry. But the primary of this line alone has a width of about $0.04 \mathrm{t}-\mathrm{m}$ at moderate intensity, hence its impurity due to this width is nearly as great as that introduced by the presence of all the satellites together.

The brighter normal and composite lines are classified in the following brief catalogue. It is based on work with an echelon by the writer. ${ }^{5}$ The structure of the lines of zinc, cadmium, mercury, thallium, sodium, and hydrogen had been previously observed by Barnes, ${ }^{6}$ Houstom,${ }^{7}$ Janicki, ${ }^{8}$ and by several earlier workers.

Lithium, sodium, potassium, rubidium, and cesium.-The brighter lines of these spectra are all composite and much alike in structure, being characterized by variable overlapping satellites. All these lines appear single; that is, the satellites all sink below the threshold of vision at low intensities. With increasing intensity two, three, or four satellites appear, broaden in situ until they overlap and blur, until when reversal proper occurs the components have all merged in continuous wings. The fainter lines of these spectra have not yet been studied.

Magnesium, calcium, strontium, barium, gold.-These lines are either all normal, or if satellites exist they are so broad and overlapping as to appear as continuous wings. Some of the calcium lines show a trace of superposed structure similar to the lines of the sodium class.

Titanium, cerium, thorium, vanadium, chromium, molybdenum, tungsten, uranium, iron, cobalt, nickel, palladium, platinum, rhodium, and osmium.-All the brighter lines of these elements appear to be normal. They were all observed up to the point of reversal,

${ }^{5}$ Astrophysical Journal, 23, p. 64-79; 23, p. 220-232; 24, p. III-I25; 1906.

${ }^{6}$ James Barnes: Astrophysical Journal, 19, p. I90-2I 2; 1906.

${ }^{7}$ R. A. Houstoun: Philosophical Magazine, 7, p. 456-467; I904.

${ }^{8}$ Ludwig Janicki: Ann. Physik, 19, p. 36-79; 1906. 
and had they been composite their structure would have been most apparent at about a tenth of that intensity. The fainter lines were not studied, but these being non-series spectra, there is every reason to expect that the fainter lines are also normal.

Manganese.-This spectrum is very remarkable. Twelve bright green lines are composite (double and triple) while all others are normal. As the arc fluctuates, the normal lines flash out and reverse while the composite lines, with their components fixed in position, remain as steady as though painted.

Copper.-The two yellow and two of the group of three green lines are composite (double or triple) while the third green line $\lambda_{5}$ I04 appears to be normal. The three composite copper lines are of a type that frequently occurs. One satellite is nearly as strong as the primary, of nearly constant relative intensity, and at a distance of about o.I t-m from it. The second satellite is on the opposite side of the primary, about O.I $3 \mathrm{t}$-m distant, hardly a tenth as bright and very variable in relative intensity. Hence the same line may appear either double or triple under slightly different conditions. A similarly variable triple structure in cadmium $\lambda 5086$ has been shown ${ }^{9}$ to be the cause of the discrepancy between the wave length values determined by Hamy and by Michelson. Hamy's electrodeless tube gave a triple line, while in Michelson's tube with electrodes the second satellite was not apparent.

Silver.-All the five prominent silver lines are composite, $\lambda{ }_{54} 6_{5}$ being quadruple and the others triple, similar to the copper lines but more symmetrical.

Indium.-The prominent blue indium line appears to be normal, the doubling observed being a case of simple reversal.

Zinc, cadmium, mercury, thallium, tin, lead, and bismuth.-All the prominent lines of these spectra are composite. The zinc red and three blue lines are triple, resembling the composite copper lines. The red, green, and blue cadmium lines are similar triplets. The seven prominent visible mercury lines are all complex and all different in structure, with from two to five satellites. $\lambda_{5769}$ is a triplet resembling those of copper, cadmium, and zinc, but more compact. Thallium $\lambda_{535} \mathrm{O}$ is a very peculiar triplet. Tin $\lambda_{5} 6_{3} \mathrm{I}$

${ }^{9} \mathrm{C}$. Fabry: Astrophysical Journal, 19, p. I I6; I904. 
and $\lambda 45^{2} 5$ are close triplets, lead $\lambda 6002$ and $\lambda 4058$ are broad, diffuse triplets, bismuth $\lambda 4722$ has two sharp, bright satellites and one faint and diffuse.

Aluminum, boron, beryllium, carbon, silicon, phosphorus, arsenic, and antimony give no visible lines of sufficient intensity to permit of a visual study of their structure.

Oxygen, nitrogen, sulphur, selenium, tellurium, chlorine, bromine, and iodine possess only faint visible lines in their primary spectra, while the lines of their secondary spectra are all over $0.2 \mathrm{t}-\mathrm{m}$ broad, and hence useless as monochromatic sources. The lines of the hydrogen primary spectrum are likewise too faint, while the secondary lines are not only broad but complex.

Helium has seven prominent visible lines that promise great usefulness as sources. Four of them have companion lines about half a tenth-meter distant, it is true, but these are relatively so faint as to be barely perceptible in an interferometer. The fact that a helinm lamp improves rather than deteriorates with use is an immense advantage. All the helium lines are fairly narrow at moderate intensity ( 0.05 to $0.09 \mathrm{t}-\mathrm{m}$ ). The relation of width to intensity will be taken up farther on.

Argon, neon, krypton, and xenon have not yet been investigated as to line structure and give little promise of usefulness, with the possible exception of the xenon red line.

There are then available two quite distinct classes of monochromatic sources, one class of fixed and the other of arbitrary purity.

I. Normal lines whose purity may be increased almost indefinitely by decreasing the density of the luminous gas and the intensity of its excitation.

2. Composite lines whose purity is high but in general can not be varied greatly. If all components remained fixed in position and relative intensity the purity could not be varied at all. Visual purity may always be increased by a decrease in intensity sufficient to lower the luminosity of fainter components below the threshold value.

The purity required of monochromatic sources for varions purposes is roughly as follows. For ultimate length standards the impurity should not exceed one part in ten million, while the center of luminosity should be known to one part in one hundred million. It is very doubtful whether spectroscopists will ever be 
able to produce a source of greater purity than this or whether other sources of error, for instance, those due to temperature variations, will ever be reduced to such an extent that greater purity will ever be necessary.

Interferometry requires ordinarily a purity of one part in one million and a knowledge of the center of luminosity to one part in ten million. Reference standards of wave length should be pure to one part in five hundred thousand and permit of the measurement of the position of their centers to one part in five million or, roughly, o.oor t-in. Refractometry and polarimetry require only a low order of purity. One part in ten thousand is not too great an impurity for ordinary work.

To obtain the highest obtainable purity, then, it is necessary to choose from normal lines. Obviously the best normal lines to choose are those which (I) have the least minimum width and (2) which increase in width least rapidly with increasing intensity and density of the radiating gas. It was to determine which normal lines have the least minimum width and intensity coefficient that the following investigation was undertaken.

\section{THE IMPURITY OF NORMAL SPECTRUM LINES.}

Since the width of a normal line varies greatly with its intensity, apparatus was so arranged that the intensity of each line could be determined by a spectrophotometer at the same time that the width was being measured with a micrometer echelon. A wide slit near the source screened off all of it except the central portion, which was radiating toward the echelon. A Nernst lamp served as reference standard. This was eventually calibrated by means of a Rubens thermopile placed in the ocular of the spectrophotometer.

'The width of a normal line appears to depend, aside from the pressure, upon the intensity alone. When, for example, copper is fed into an arc with carbon electrodes, the line 5106 is of the same width at a given intensity as when copper electrodes are used. It is the same whether metallic copper or a copper salt is fed into a carbon arc. If, as the salt burns away, the current is increased to bring the intensity of a line up to its original value, its width also is restored. Nor is this ratio of width to intensity appreciably affected by the addition of an alien salt or metal to an arc. A line 
has less diffuse wings when produced in an oxyhydrogen flame than when produced in an arc, but it appears to possess the same mean width at a given intensity in the two cases.

\begin{tabular}{c|c|c}
\hline Slit Reading & Line Width & Slit Width \\
\cline { 2 - 3 } 5.4 & $121 \mu$ & $104 \mu$ \\
5.3 & 110 & 94 \\
5.2 & 98 & 84 \\
5.1 & 91 & 74 \\
5.0 & 79 & 64 \\
4.9 & 67 & 54 \\
4.8 & 53 & 44 \\
4.7 & 39 & 34 \\
4.5 & 26 & 24 \\
4.5 & 15 & 14 \\
4.4 & (faint) & 4 \\
4.35 & slit closed & 0 \\
\hline
\end{tabular}

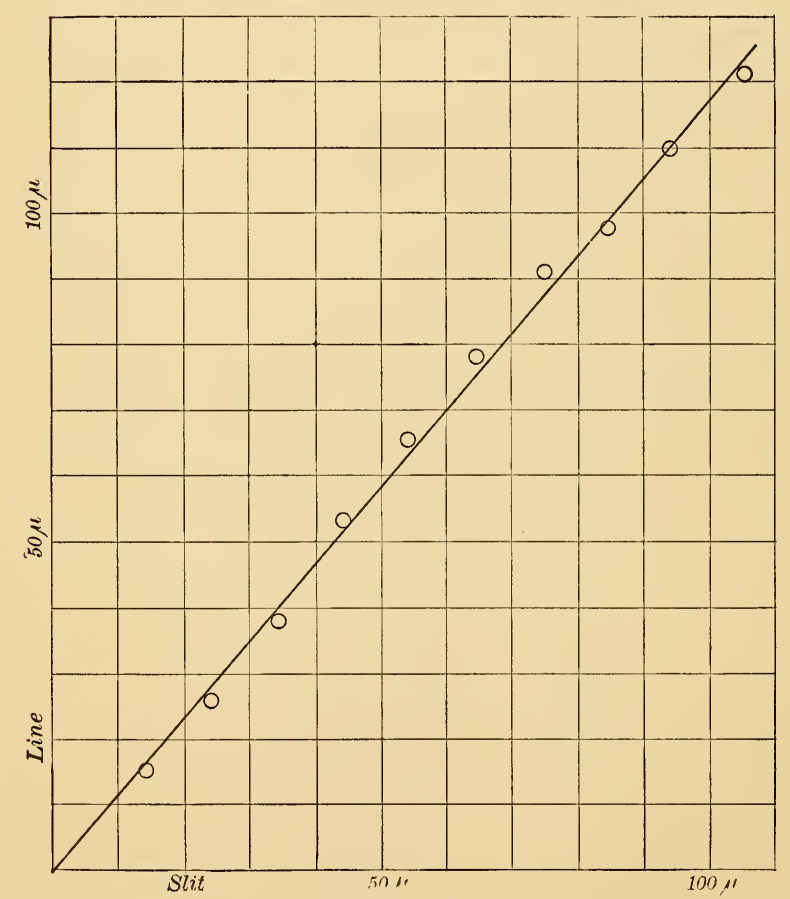

Fig. 1.

The green mercury line has three sarellites on the side of the primary toward the red. These three lines are the narrowest bright lines known to the writer, and the inner one of these was for this 
reason chosen to verify the correction for slit width to be applied to the measured width of a line. The figures quoted above and the points on the accompanying graph are from the first set of readings taken.

Line width and slit width are seen to be proportional to each other far within the possible errors of measurement. The resolving power of the echelon is seen to be considerably (at least five times) greater than the calculated value of o.or tenth-meter. The actual width of the line itself is from the plot certainly not greater than one micron or almost $0.00 \mathrm{I} \mathrm{t}-\mathrm{m}$ in terms of wave length. In other words, the light of this satellite of the green mercury line is homogeneous to within one part in at least five million. The main primary of this line is fully twenty times as broad as this satellite while the whole line, measured from extreme satellites, is three hundred times as broad or about $0.3 \mathrm{t}-\mathrm{m}$.

The results of some observations on the relation of width to intensity of the yellow helium line $\lambda 5876$ are given below. They are the first and last of a series of observations. The helium was contained in short, stout Plücker tubes excited by means of a 5,000 volt transformer. The intensity was varied by varying the current in the primary. The two sets of observations quoted were made more than a week apart on two different Plücker tubes. The echelon slit was open I4 microns in one case and 24 microns in the other, the widths of the line being corrected by means of the plot reproduced in Fig. I above.

\begin{tabular}{|c|c|c|c|c|c|}
\hline \multicolumn{3}{|c|}{ Tube I, Slit Width $24 \mu$} & \multicolumn{3}{|c|}{ Tube 2, Slit Widtin I $4 \mu$} \\
\hline \multirow{2}{*}{ Intensity } & \multicolumn{2}{|c|}{ Line Width } & \multirow{2}{*}{ Intensity } & \multicolumn{2}{|c|}{ Line Width } \\
\hline & Linear & Wave Length & & Linear & Wave Length \\
\hline 1.0 & $42 \mu$ & $.046 \mathrm{t}-\mathrm{m}$ & 0.85 & $40 \mu$ & $.044 \mathrm{t}-\mathrm{m}$ \\
\hline 1.9 & 47 & .052 & 1.44 & 46 & .050 \\
\hline 3.2 & 57 & .062 & 2.6 & 56 & .062 \\
\hline $4 . \overline{7}$ & 66 & .072 & 3.9 & 64 & .071 \\
\hline 6.7 & 76 & .084 & 5.0 & 69 & .076 \\
\hline 9.5 & 93 & .102 & 6.0 & 72 & .079 \\
\hline 12.9 & 109 & .120 & 7.1 & 75 & .083 \\
\hline \multirow[t]{3}{*}{19.0} & 115 & .126 & 9.0 & 77 & .085 \\
\hline & & & 12.2 & 90 & .099 \\
\hline & & & 20.2 & 115 & .126 \\
\hline
\end{tabular}


The width of the line is seen to vary considerably with its intensity, more than doubling within the range available. The relation between width and intensity is approximately linear for low and moderate intensities. At great intensities the width is, if anything, less than this proportionality would indicate. At the intensity called to the capillary of the tube was just commencing to burn and show sodium lines, and possibly this might account for the falling off from the linear relation at high intensities, but arc lines show

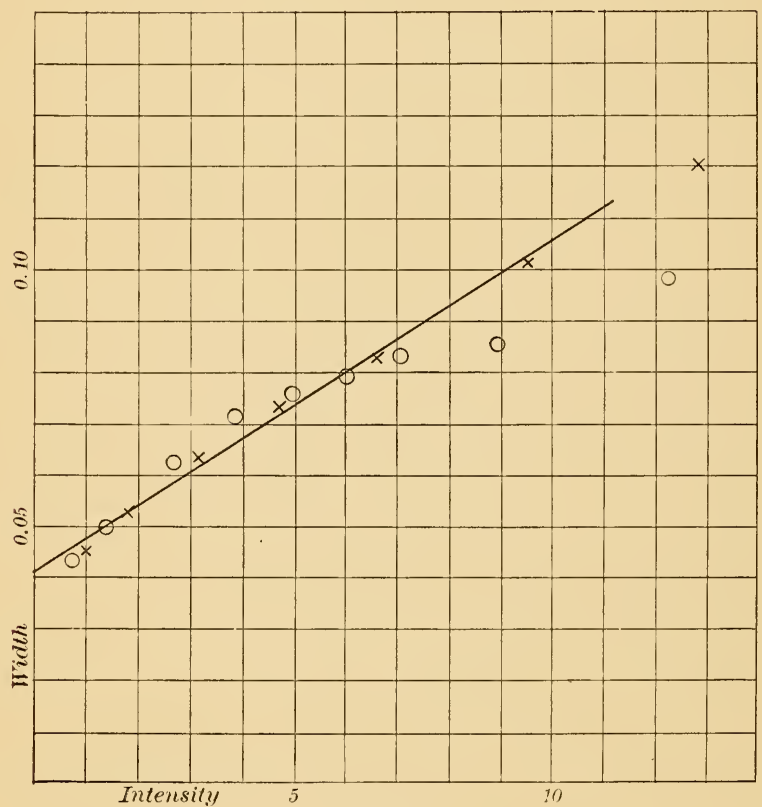

Fig. 2.

Width Intensity. He $\lambda 5876$.

a similar falling off. Plotting against a quadratic or logarithmic abscissa does not help matters.

Extrapolating back to zero of intensity, there is good evidence that the line then possesses a finite width of perhaps $0.040 \mathrm{t}-\mathrm{m}$. Visual observation tends to confirm this, for at intensities much lower than the lowest at which the width can be measured with any accuracy, the line still appears as a ribbon of light rather than as fine line. The appearance of the line suggests that it is an unre- 
solved composite. With exceptionally favorable conditions I have seen a faint separation into two components. If the line had two components, each of zero width, this limiting width of $0.040 \mathrm{t}-\mathrm{m}$ would represent the distance between their centers.

A few normal arc lines are sufficiently isolated in their spectra and variable in intensity to permit of fairly accurate observations of the width-intensity relation being made. Arcs of from two to eight amperes were used. As the arc fluctuated the maxima and minima were found to be reasonably cunstant in intensity and to permit of measurement. The best of the results are given below in the form of linear equations between width $\mathrm{W}$ and intensity $\mathrm{I}$. They are taken from the plotted curves, all of which were of the same general shape as that reproduced in Fig. 2. W is expressed in tenth-meters and $I$ in terms of an arbitrary unit such that the intensity of the bright green line Fe $\lambda 5328$ from an iron arc of 5 amperes in open air is about 0.5 .

$\begin{array}{lll}\mathrm{Ba} & 6342 & W=0.034+0.0030 I \\ \mathrm{Ni} & 5477 & W=0.032+0.0035 I \\ \mathrm{Fe} & 5328 & W=0.025+0.0045 I \\ \mathrm{C} 11 & 5106 & W=0.033+0.0030 I \\ \mathrm{Fd} & 5296 & W=0.032+0.0040 I\end{array}$

for comparison may be added

$\mathrm{He}$

58,6

$W=0.040+0.007 I$

$\mathrm{Hg} \quad 546 \mathrm{I}$ satellite $W \quad$ o.0oI

The results for the arc lines show a striking uniformity both in limiting width and in intensity coefficient. Other lines gave data so similar and the accidental errors were so large that it was found to be only duplicating work to take measurements on them and only minimum width and intensity at reversal were recorded. With the arrangement used (echelon slit perpendicular to the spectrum lines formed by the preliminary dispersing apparatus) many lines were simultaneously in the field of view, so that if any of the many hundred lines observed had possessed exceptional qualities they would have been at once noticeable. 
The minimum width of all normal arc lines produced at atmospheric pressure lies between $0.02 \mathrm{t}-\mathrm{m}$ and $0.05 \mathrm{t}-\mathrm{m}$. This width is of the same order as the width to be expected from the Doppler effect and the kinetic gas theory. For, if $\delta$ is the spectral width of a line produced by a source moving with a mean speed of $u \mathrm{~cm} / \mathrm{sec}$, then if $V$ is the velocity of light, $\delta: \lambda=2 u l V$. For air at room temperature and pressure, $u$ is usually taken as about $4.5 \times 10^{4} \mathrm{~cm} / \mathrm{sec}$, increasing with the square root of the temperature. Hence the width of a green line should be at least $\delta=5000 \times 2 \times 4.5 \times 10^{4} 3 \times 10^{10}=$ $0.015 \mathrm{t}-\mathrm{m}$. If the arc gases are at a temperature of $3000^{\circ}$, then this width should by the kinetic theory be multiplied by the factor $(3000300) \frac{1}{2}=3.16$, hence $\delta=0.015 \times 3.16=0.047 \mathrm{t}-\mathrm{m}$. Similarly for red line at $7000, \delta=0.034 \mathrm{t}$-m.

Reversing the argument, the widths observed indicate that only the thermal motions of the radiating particles are concerned in the broadening. The conduction of a current by the gas in the arc does not add greatly to the translatory motions which the particles of a gas would have in virtue of their high temperature alone. In other words, line-of-sight motions are unaffected by the current through an arc.

At low pressure an enclosed arc with electrodes of iron, copper, brass, and nickel were used. With nickel some observations were obtained with a clear window without disturbing the photometric adjustments used in the previous work. For Ni $\lambda_{5477}$,

$$
W=0.006+0.007 I
$$

with a large probable error on account of the great fluctuations and altered character of the arc flame. Other lines were observed to have roughly the same minimnm width. From kinetic theory the width at I $\mathrm{cm}$ pressure should be $\left(\mathrm{I}^{\prime} 76\right)^{\frac{1}{2}}=0 . \mathrm{I}$ I of the width atmospheric pressure if arc vapors remain at constant density. The latter for $\lambda 5477$ at $3000^{\circ}$ is $0.043 \mathrm{t}-\mathrm{m}$, hence at I cm pressure should be $0.0047 \mathrm{t}$-m, considerably less than the width observed.

The developinent of a typical normal line is then somewhat as depicted in Fig. 3, where frequency (or wave length) is plotted against intensity. At high pressures, say in an open air arc, the development of the line is shown by the full lines, at low pressures 
by the dotted lines. The intercept $\dot{a} b$ on the frequency axis represents the minimum width of impurity of the spectrum line at a very low intensity. With increase of intensity the line broadens and finally separates into two as the intensity corresponding to the point $c$ is reached. With further increase in intensity the two components continually broaden and separate. At low pressure the minimum width is less and the line twins at a lower intensity.

There appears, then, to be little choice between various normal lines so far as minimum width and intensity coefficient are concerned. Normal lines certainly do not differ by as much as a factor of ten in these properties. There being so little diversity leaves a wide range of lines equally available. The green nickel line is perhaps as easily produced and segregated as any other.

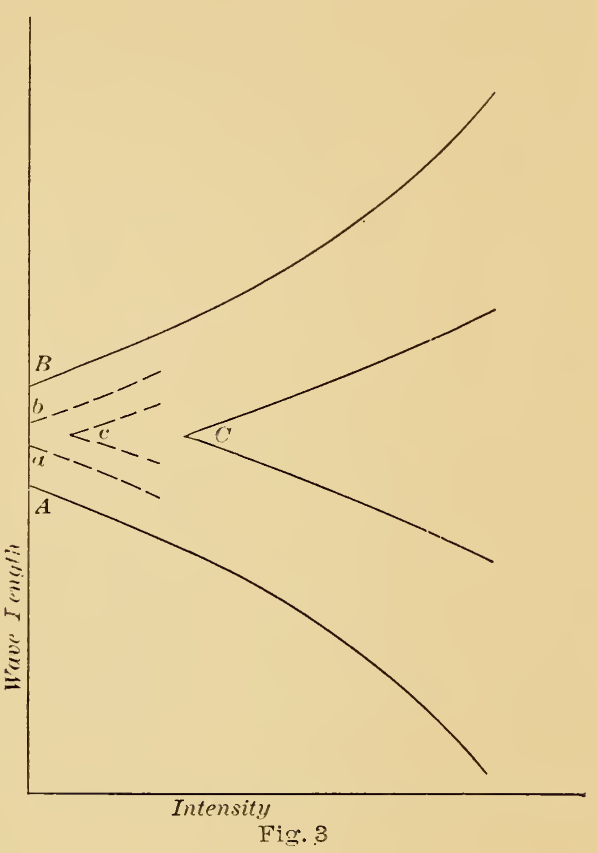

If a number of lines of the same energy intensity, but in different parts of the spectrum are available, it is of interest to know which will be best for visual use. In this case their relative luminosity will be given roughly by the function,

$$
V=e^{-\pi(\lambda-5 \cdot 1)^{2}}
$$

for an arerage observer, and their relative visual brightness by the logarithms of these values. This function has been deduced from a great number of observations by different investigators. Numerical values of this function are given below:

$$
\begin{aligned}
& \lambda=\left\{\begin{array}{lllllllllll}
520 & 530 & 540 & 550 & 560 & 570 & 580 & 590 & 600 & 610 & 650 \mu \mu
\end{array}\right.
\end{aligned}
$$

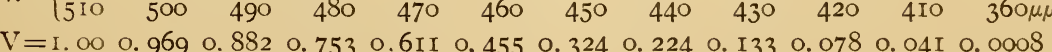


In conclusion, the character of some of the purest known sources of illumination may be summarized:

I. The most nearly homogeneous light sources yet studied are some of the satellites of the composite lines. Three satellites of the green mercury line are homogeneous to within one part in at least five million. These are, however, not yet available for interferometry because their segregation involves the use of an echelon grating. These satellites appear to be free from the Doppler effect, but this point is not yet well established.

2. Second in homogeneity at moderate intensity are the normal arc lines. These are all roughly $0.05 \mathrm{t}-1 \mathrm{~m}$ broad at atmospheric pressure, and hence possess a specific impurity of about one part in five-hundred thousand. Produced at low pressures these normal arc lines are approximately $0.006 \mathrm{t}$-1n broad, with a specific impurity of one part in four million.

3. Of third rank as to purity are the composite lines produced in vacuum tubes measured between extreme components. These have a width varying from $\mathrm{O}$. I to $0.3 \mathrm{t}-\mathrm{m}$, or even more.

The purest of these, namely, the zinc and cadmium lines, have a specific impurity (as defined in this paper) varying from one part in a million to one part in sixty thousand. 